\title{
Quartz Micromass Balance as a sensor for Chemical Warfare Agents
}

\author{
K.D. Vyas, Joint. Director, \\ Defence Research And Development Establishment \\ (DRDE), DRDO, Ministry of Defence, Jhansi Road, Gwalior \\ E-mail : aswkdv@yahoo.co.in
}

\section{Abstract}

Chemical warfare agents are deadly toxic and their even one gram quantity can kill around hundreds of soldiers. Such chemicals are potential threats, for civil population, if used by terrorists, as these toxic agents are colorless, odorless, highly vaporizable, and can be dispersed easily in air. For protection, detection of such chemical vapours at extremely low concentration (Sub PPM Level) is necessary. DRDE, Gwalior is a pioneer DRDO Lab, doing R \& D for chemical detection and protection, against chemical warfare Agents, and has developed a large number of technologies, for detection as well as protection from CWA threats. Quartz Micromass Balance (QMB) works on the principle of piezo electric effect. ${ }^{1}$ A differential quartz crystal oscillator made from Quartz crystal, coated with chemically active interface, provides a reversible, and rapid detection signal for sub PPM level concentration of toxic chemical agent Sarin and its simulant DMMP. Quartz Micromass Balance sensors are quite sensitive and rapid. The science associated with such a sensor, its functional and design aspect and representative practical results are being described. DRDE, Gwalior has developed a large number of technologies for NBC protection of the armed forces from a NBC (Nuclear, Biological and Chemical) attack. For protection, Face mask Canisters, based on active charcoal filtering have been made, which can absorb toxic chemicals, biological bacteria, \& spores, radioactive dust particles etc and provide breathable air in a toxic chemical environment. Such filtering canisters and high volume filers are in use of Indian forces for civil preparedness.

Key words : Quartz Micromass Balance, PPM level, Piezo electric effect

\section{POTENTIAL TOXIC AGENTS ARE OF FOLLOWING TYPES}

(1) Nerve Agents : These include chemicals named Sarin, Soman, Tabun, and Vx, which at around 2 PPM concentration can kill the person. Death occurs due to paralysis of respiratory system.

(2) Blister Agents : these include, chemicals known as Sulphur Mustard and Nitrogen Mustard, which are viscous liquid, and their few PPM concentration by contact, generates painful blisters on skin.

(3) Blood Agents : These include chemicals like hydrogen cyanide, whose around 10 PPM concentration is deadly toxic.

(4) Choking Agents : These include agents like Phosgene gas around 20 PPM concentration is deadly toxic.

Some of the above agents e.g. phosgene gas was used in World War-II. Also reports of use of Sarin, in the terrorists attack and mass casualties are available. For protection against such chemical vapours, face masks are to be put on and protection suits are to be worn. However if suitable sensitive detectors are not available, soldiers will inhale chemical agents, before they take any protection. Thus early detection of a chemical agents, at very low concentration is extremely necessary.

Sensor instruments for sensing chemical agents have been made based on various following principles:

1) Ion Mobility Spectrometry
2) Gas Chromatography

3) Surface \& BulkAcoustic wave based

4) Flame Photometry based.

While IMS, GC, \& FPD based sensors are now commercially available, they are very costly and possess false alarm upto some degree due to cross sensitivities. Cost of IMS sensor varies from Rs. 5 Lakhs to 10 Lakhs each. GC \& FPD sensors are also available at around 5 to 7 Lakhs. Thus for making a reliable network of multiple sensor to cover a wide area for monitoring, above sensors are too costly, to be employable for civil population.

Sensors working on bulk acoustic waves are designed using quartz micromass balance. Quartz crystal wafers, AT cut, were being used since long for making crystal oscillators. While crystal oscillators, possess highly stable frequency, the same can be varied based on the mass deposited on the crystal surface. These wafers, are commercially available and can be used for making QMB sensors.

\section{QMB SENSING MECHANISM}

The vibrational frequency of a piezo crystal decreases on adsorption of foreign substance on its surface. The variation of the frequency $(\Delta f)$ with mass deposited $(\Delta \mathrm{m})$ is governed by the Surbrey equation as described below

$\begin{aligned} \Delta \mathrm{f} & \left.=-2.3 \times 10^{6} \mathrm{f}_{0}{ }^{21} \Delta \mathrm{m} / \mathrm{A}\right) \\ \text { Where, } A & =\text { Crystal surface area in } \mathrm{cm}^{2}\end{aligned}$




$$
\begin{array}{lll}
\mathrm{f}_{0} & = & \begin{array}{l}
\text { resonant frequency of } \\
\text { crystal oscillator in } \mathrm{MHz}
\end{array} \\
\Delta \mathrm{f} & = & \text { change in frequency in } \mathrm{Hz} \\
\Delta \mathrm{m} & = & \text { mass deposied in gms }
\end{array}
$$

For a crystal of resonance frequency $10 \mathrm{MHz}$, and surface area about $0.23 \mathrm{~cm}^{2}$ frequency change is about 1 $\mathrm{Hz}$ for $10^{-9} \mathrm{gm}$ deposition of mass.

\section{TRANSFORMATION OF CRYSTAL OSCILLATOR AS CHEMICAL VAPOUR SENSOR}

Simple piezo crystal based oscillator cannot sense chemical vapours. However when a thin film of sensing material is coated on the crystal surface, the oscillator becomes chemically active. The coating materials are tailor made polymers which can reversibly adsorb the gaseous material. The sensitivity, response time and recovery time of the chemical sensor depend upon the properties of chemically active material or chemical interface. Uniformity of the chemical layer formed and film thickness also affects the sensitivity, response and recovery time.

As crystal oscillators are sensitive to some degree of temperature and pressure variations, differential crystal oscillators ${ }^{2}$ are used so that effect of temperature and pressure variations are compensated. Schematic Block Diagram of one channel differential crystal oscillator is as shown below in Fig. 1.

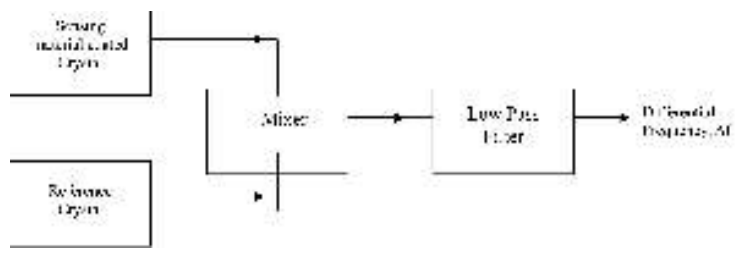

Fig. 1. Block diagram of single channel differential crystal oscillator

\section{CHEMICAL INTERFACES}

Chemical interfaces for Quartz Crystal sensors are selected based on the inherent adsorption properties of a variety of compounds. Research work is going on to select suitable chemical interfaces for various types of chemical vapours. Large numbers of chemical interfaces have been reported by various researchers. Some of the chemical interfaces for nerve agent Sarin and Blister Agent Sulphur Mustard reported in open literature are as described below:

\section{Sensing polymer for sarin :}

1) Collodian

\section{2) Fluoropolyol \\ 3) Ethylene Diamine Copper Butyrate}

\section{Sensing polymer for sulphur mustard}

1) Poly epichlorohydrin

Quartz crystal wafer on its both sides is coated by Gold Electrodes. Chemical interfaces are coated on crystal on electrode surfaces in a very small quantity i.e. 2 to 4 micro gram, employing either spin coating, or solvent evaporation or Air brush technique. Coated crystals are annealed at suitable high temperature to desorb the solvent. The type of solvent used also affects the sensitivity of the transducer formed. Coating process is optimized to get the maximum sensitivity from a piezo transducer. Thus open piezo electric crystal, by interface material coating is converted into a suitable piezo transducer for chemical vapour sensing.

As coated crystals may have some degree of cross sensitivity to ambient humidity, and other Lab solvents. It is desirable to use array ${ }^{3}$ of 03 or more crystals in a smart vapour sensor. The output of all the piezo transducers is fed to a microcontroller, which by employing pattern recognisition ${ }^{4}$, provides specificity to the chemical vapour sensing.

\section{SMART CHEMICAL VAPOUR SENSOR}

Schematic Block Diagram of a Smart sensor is as given below. It has various following parts, in Fig 2.

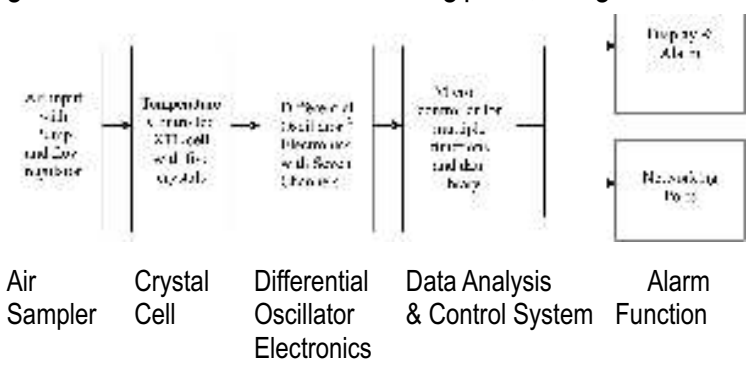

Fig. 2. Schematic block diagram of a smart QMB sensor

\section{Air sampler}

This comprised of smooth air flow pumps, equipped with dust filter, draws ambient air, and sends in crystal cell. Samplers do have, pre heaters, air regulators, and temperature sensors for improved performance. Air sampler with pre concentrators are also employed

\section{Crystal cell}

Crystal cell is usually made by inert, non adsorbing material, having minimum possible volume and least dead space. The cell has low thermal mass so that its temperature is easily regulated. Outlet of the sampler is 
connected to inlet of the crystal cell, in which incoming air, after passing from crystal goes to outside environment. Temperature of crystal cell is regulated around 20 to 30 degree Celsius, where in adsorption process is prominent. Peltier coolers are also mounted on crystal cell where ever, instrument requires high temperature operation i.e. at around $50^{\circ} \mathrm{C}$.

\section{Differential oscillator electronics}

Crystal oscillator can be made using Operational Amplifier with R C coupling having Quartz Crystal in feed back loop. However for better stability CMOS series NAND gate are utilized to make crystal oscillators. Oscillator outputs are mixed using Exclusive OR Gate ant output is isolated using a capacitive low pass filter. Schematic diagram of two channel oscillator circuit is as shown below in Fig. 3.

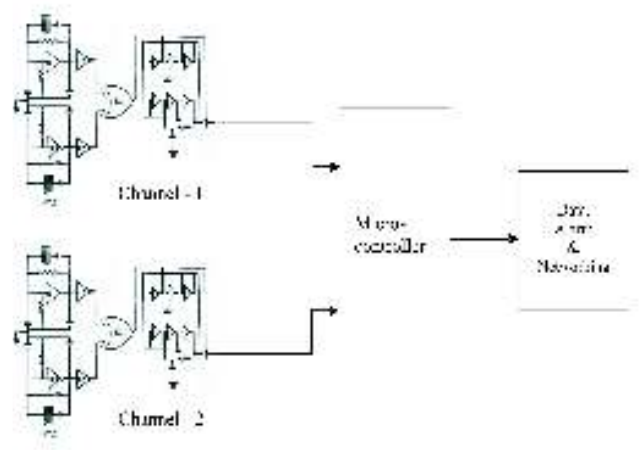

Fig. 3. Two channel oscillator circuit

\section{Data analysis and control system}

This part generally uses a microcontroller with suitable memory size to handle, data storage and pattern recognition functions. Microcontroller input ports are given, differential frequency, inputs, which are counted and denoted. The differential outputs, initially taken in clean air serve as baseline. For every oscillator base line frequency may be different. Now when the instrument input is exposed to the chemical vapours, of different concentrations, differential oscillator frequency increases, which serve as the sensing signal.

Pattern of 3 or more piezo crystal sensors are recorded for various gases, individually, as well as gas mixtures of various concentrations. The data of crystal cell temperature, and input gas temperature \& Relative humidity are also recorded. Recorded data are analyzed for pattern recognition using principal component analysis or Neural network, or any other suitable approach and, data library of pattern models, for particular applications is built. Such type of data analysis add specificity to the sensing and isolate unwanted interferences. In addition to these software is also equipped with diagnostic and control functions.

\section{Alarm function}

Above type of sensor described is a point sensor and can sense chemical vapour in near vicinity. However alarms could be audio or video to warn the user regarding chemical threats.

\section{Network of sensors}

Wired or wireless network of many such sensors, make a system capable of wide area monitoring or monitoring of a closed space depending upon design configuration. Since well-designed QMB sensors could be cost effective, such networks will be highly economical compared to many other types of sensors (IMS or FPD sensors) and can provide cost effective solution for wide area sensing of CWA threats.

\section{Experimental example}

DRDE, Gwalior has developed Quartz crystal based chemical vapour sensor. We have employed solvent evaporation technique for crystal electrode surface coating. Collodian polymer has been used as chemically active interference material. the quantity of material loaded was around 4 micro gram.

The instrument was tested with Nerve agent simulant DMMP (Dimethyl methyl phosphate). Crystal was housed in a crystal cell made of glass and vapour of test chemical was delivered using a standard vapour generator. Vapour concentration was $1 \mathrm{mg} / \mathrm{m}^{3}$ i.e. $=0.02$ PPM approx, calibrated by Gas Chromatograph.

The output was recorded as sample and purge cycles of differential frequency shift, using a online, networked PC. A print copy of the response cycle of sensor output is as shown below in Fig. 4 .

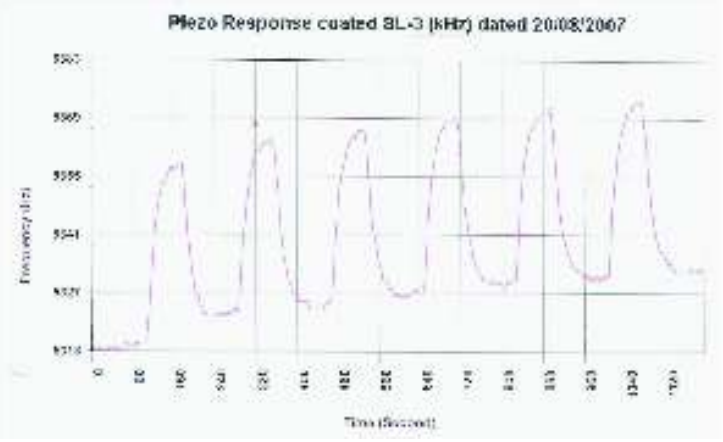

Fig. 4. Graph of response cycle of sensor output

By observing the response cycle it is evident that response of the instrument is reversible. Its response time 
is less than 30 seconds and also recovery time is below 1 minute.

This type of single channel Quartz crystal sensor, sustains the crystal sensing output for more than a year. Thus the sensing element will need only periodic replacement comprising of a period beyond a year.

The above observation indicates that, using multi channel, design of the above type of sensor may produce a capable sensing system for chemical vapours. Also these sensor may find numerous applications for various types of industrial gases, where bulky sensors design based on other techniques are being employed.

\section{CONCLUSION}

Strategic importance of NBC detection has been described and the quartz crystal; micro mass balance (QMB) technology for chemical vapour sensor has been discussed. Experimental results from, a QMB sensor, designed for Nerve agent simulant, in DRDE were also described.

\section{REFERENCES}

[1] J Niavay and G.G. Guilbailt,Nov 1977, Application of the piezoelectric crystal in Analytical Chemistry, Vol 49, No. 13, P. 1990.

[2] Mathew Michalski Nov 1994, Dual QMB Oscillator Circuit, Analytical Chemistry, Vol66, No. 11, P. 1847

[3] U. Schramm et. Al., 2000, A QMB based Ammonia Sensor for humid air, Sensors and Actuators , B 67, 219 to 226

[4] Tim Hamcher et.al. 2003, Measurement of odorous gases close to odour threshold with QMB sensor system with an integrated pre-concentration unit. Senosrs and Actuators B 95, 39 to 45.

[5] Sushanta Banerjee, Krishna Dutt Vyas, et. al. 1996, Quartz Piezoelectric crystal Crystal Detector used for sensing organo-phosphorous compound. Journal of Polymer Materials, 29-33 